\title{
The MRAMOR Workstation
}

\author{
A.A. Baehrs \\ A.P. Ershov Institute of Informatics Systems, SB RAS \\ baehrsaiis.nsk.su
}

\begin{abstract}
The paper describes the experience of creating the MRAMOR workstation in 1980-1987, a quality workspace for the publishing business built on a weak element basis. The aim of the work consisted in the creation of hardand-software foundation and a system of workspaces for professional publishers. This accounted for the initially complex approach to the problem, which combined hardware, software, font, and visual design. We produced a pilot batch of forty workspaces, basic program software for the station, and application software for workspaces of professional publishing systems for electronic publication preparation. These convenient and highly effective workspaces went into test operation and they served to produce a large number of publications of high polygraph quality.
\end{abstract}

Keywords: Workstation, electronic publishing, parallel complex development of software and hardware.

\section{Introduction}

This paper dwells upon the experience of the creation in 1980-1987 of the MRAMOR workstation, a quality workspace for the publishing business built on a weak element basis. The project, dubbed "The RUBIN (RUBY) project for the PRAVDA newspaper", was part of international cooperation between the USSR and the People's Republic of Poland. The Soviet side was represented by the Pravda publishing office, the SB AS USSR Computing Center, and the M.V. Keldysh Institue of Applied Mathematics, while the MERA-Blone precision mechanics factory and the COBRESPU-Center of television equipment-represented Poland. The RUBIN project was part of the list of key research projects of the GKNT USSR (Gosudarstvennyj Komitet po Nauke i Technike, USSR State Commission on Science and Technology) in the years 1980-1985.

An academic institute developed the architecture, design, and functional characteristics of the MRAMOR workstation and of the MRAMOR-based workspaces, as well as its basic and application software. The design group at the MERA-Blone factory performed the realization of the developed ideas into working machinery and for preparation for serial production. The Pravda publishing office substantially supported the work within the RUBIN project.

The developers' collective consisted of the following teams:

The Laboratory of Experimental Informatics of the Computing Center of SB AS USSR was represented in the MRAMOR WS project by A.A. Baehrs (GEO), Yu. 
Bovkun, A. Kovalenin, A. Melnik, A. Mullagaliev, G. Nesgovorova, E. Ovcharenko, V. Polyakov, S. Rudnev, M. Sadomskaya and V. Chetvernin.

Our polish colleagues at the MERA-Blone factory were M. Augustinyak, J. Zawadsky (SEO), J. Zagraek, A. Kolodeyak, Z. Luchuk, J. Matrash, T. Moshevich, R. Pacek and S. Shumsky. The Warsaw Center of television equipment (COBRESPU) under the direction of J. Kania and L. Nepekla developed the video displays for the MRAMOR WS. All current organizational tasks of the RUBIN were the job of the technical department of Pravda publishers, and a personal duty of their leader, V.A. Tiefenbach.

The author, who was the general executive officer and chief designer of the project, considers it a pleasant duty to mark the exceptionally friendly and creative interaction of all members of this international collective, consisting of specialists from many fields. I am still cordially thankful to all the members of this interesting and long work. Additionally, I wish to acknowledge the care and attention the project received from our teacher, academician Andrei Petrovich Ershov.

\section{The Project}

The stages of production process research in the Pravda publishing office and of system analysis of these processes preceded the start of the RUBIN. The results of the research, summed up in the "General Scheme of Creation and Development of the RUBIN system for the PRAVDA Newspaper" and approved by the Pravda editorial board in February of 1979, held that the system would consist of a peripheral network and a central computational complex (CCC). The CCC, with a large information and reference database for analysis and verification of published materials and perspective planning of newspaper issues, appeared on the older models of ES computers. We created the MRAMOR workstation as the terminal base of the peripheral local network of workspaces for the publishing office employees. It was necessary to automate laborious editing and publishing processes to make it possible to process large volumes of textual information in strictly limited time, which is of crucial importance in newspaper publishing.

The aim of the work consisted in the creation of hard-and-software foundation and a system of workspaces for professional publishers. This accounted for the initially complex approach to the problem, which combined hardware, software, font, and visual design.

We produced a pilot batch of forty workspaces, basic program software for the station, and application software for workspaces of professional polygraphic systems for electronic publication preparation. These convenient and highly effective workspaces went into test operation and they served to produce a large number of publications of high polygraph quality. These workspaces included regular publication of the ENSK city newspaper, preparation of a number of issues of Nauka and EKO magazines, and a large number of books.

Serial instances of MRAMOR were shown at the "Siberian Device 87" exhibition (Akademgorodok, 1987), where the design received the Diploma for the original solution, at the All-Poland POLIKON-87 Conference (Poznan, 1987), and at "MERABlone Factory - to Soviet Informatics" exhibitions in Moscow, 1987, and Vilnus, 
1988. At the $1988 \mathrm{SB}$ AS USSR projects contest, the MRAMOR received the Diploma of Third Degree award.

\section{Main Features}

The inspection of the Pravda publishing and printing offices in 1977, the results of which were presented in the report called "System analysis of production processes of the publishing of Pravda newspaper" allowed to highlight the main channels of information processing and their substantial characteristics necessary for the creation of RUBIN. These are as follows.

○ processing of texts, typesetting, make-up and preparation of newspaper typing range print forms

o planning and management of issues and publication

$\circ \quad$ information and reference service and proofing of published facts

Various workspaces for editors, publishing and printing office employees must provide cooperative coordinated access to these three streams, realization in various combinations (defined by the workspace type) of the needed applied functions, and a possibility to use results of one work in another.

\subsection{Considerations}

It was necessary to take into consideration several mandatory limitations stipulated by the customer and situation during the realization of the RUBIN. These included the fact that we had to build the equipment chiefly on domestically made basic elements and components. As an exception, they allowed us to use microchips, components, and devices produced in COMECON countries. This understanding was essential to retain maximally the technology of work that had been established in the editorial office and the style an interaction principles of its employees. There was an understanding that users of such social rank would disagree to adapt to inconvenient functions and features of equipment and programs.

On the other hand, one should remember that by the end of the 1970s, the computer stock in the USSR consisted mostly of ES and SM computers equipped predominantly with borrowed, so-called "States" software. They were totally unsuitable for work in the editing business. Terminal equipment acceptable for editing and publishing in preparation of publication was practically absent; the domestic Kaskad phototypesetting automata complex was morally outdated; font supplies of the phototypesetters and terminals were plainly primitive. In essence, the foreign polygraph computer systems employed at the time were poorly suited to the needs of local practice and created difficulties in publication in Russian and languages of other peoples of the USSR.

\subsection{Construction}

It was possible to make the CCC from one of the older ES models to create application software using the existing OS and DBMS. However, it was obvious that 
for a terminal system of the RUBIN level, it was impossible to solve automated workspace problems only by means of writing software for any of the existing and practically available mini- or microcomputers. Thus, we decided to create a new machine of the macro-mini class, dubbed "the MRAMOR Workstation".

Considering the available element base and form factors, it was clear that the computational powers of a single K580VM80 microprocessor would not suffice to provide the needed functionality and features of workspaces. Hence, we solved the problem we faced by parallel development of software and hardware for the new complex. We also had to keep in mind the practical limitations of the so-hardly-found production plant, the polish MERA-Blone precision mechanics factory.

\subsection{Requirements}

The development project of MRAMOR included the following requirements to the functions of the new hard-and-software complex:

o modularity of hardware, basic and application software;

o multi-processor architecture, including the possible heterogeneity of hardware and software;

o multi-tasking in serving the user with multi-program task load of processors;

o multi-seating of the workstation with connection between workspaces;

o multi-window interface, including the multi-screen feature of the workspace;

o federativity, i.e. operational interaction allowing users, processes and processors to work in group mode

o high quality of representation on the screen and in working printouts of polygraph aspects of the processed publications, including the multitude of national languages, writing types and fonts.

For all these various requirements to be realized within a common approach that would unite the possibilities of hardware and software tools and allow to make decisions on the project, a conceptual model of computational process execution organization was developed. Moreover, the peculiarities of preparation of different publications in the Pravda publishing office and the experience gained during the development of SAPFIR system for the first model-printing house provided a conceptual model of supporting the electronic preparation of publications.

These conceptual models, together with the above listed requirements, became a basis of the MRAMOR WS development, which lead to solving a number of all-new problems. These are as follows.

o Practical realization of parallel design of united whole hard-and-software environment, including equipment, basic and application software, user interface, and workspace design;

o Development of original open equipment, with heterogeneous computing blocks, and provide the necessary characteristics of workspaces even despite the weakness of the available elements, through the compensation of this weakness using of architectural and engineering solutions by means of possible choice between hardware and software realization of the needed functions; 
o Creation of computations organization model - high-level operational environments - allowing to organize parallel execution in the form of working mixture of a large number of interacting processes flowing for the purposes of several users on a multitude of virtual (including real) processors, each of which works in multi-program mode;

o Development of an operational system and software controlling the work of the multiprocessor complex on the basis of the given computational model;

o Creation of a multiprocessor system from heterogeneous components and organization of their joint work in the mode of multi-program execution of a large number of linked processes for the purposes of several users simultaneously, which would provide the necessary degree of parallelism by organizing a working mixture of virtual machines on heterogeneous computing tools.

o Creation of a possibility of further extension of MRAMOR WS functions through addition of modules, devices, and software, resulting in a wide range of workspace configurations available with MRAMOR, using a small number of produced module types.

In this way, MRAMOR embodies a number of original architectural, engineering and program solutions, some of which have become ubiquitous (multi-bus architecture with heterogeneous processors, portrait display layout, multi-window interface, chord keyboard input, and programmable fonts with different tracing of variable-width symbols).

\subsection{Architecture}

The pilot batch included two MRAMOR WS models: a two-seater and a four-seater. In addition, we produced two instances of single-seater multi-screen workstation for the needs of the developers. The computing blocks of the station develop around the 8086 processor and its coprocessors: arithmetic and input-output. The block links the system and local buses and contains schemes of 15 termination inputs, where we could connect any of them to any of the termination sources, the mechanism of page addressing, and EROM microchips with volume totaling $32 \mathrm{~K}$ of 16 -bit words.

We developed an original mechanism of addressing extension for the computing blocks: a scheme that defines the purpose of processor calls to the memory by state signals and by the ordinal number of cycle. The 16-bit address of command access, stack, or data processing was supplied with one of the three-order program-defined prefixes, stored in a special register. Thus, we split the main memory into four subspaces, any three of which one could simultaneously assign to the program as subspaces of commands, data, or stack.

The system provided page access to memory using hardware-implemented readdressing table (RAT). The processor could read or write directly any word in RAT. There are two page size variants -4 Kbytes and 16 Kbytes. The RAT generates signals directing the address either into the permanent memory or into its local bus or system bus (and further through the system bus into local buses of other blocks). In the $16 \mathrm{~Kb}$ variant, it can use all bits of processor address, the 14 lower ones defining the page shift. 
In the result, the processor has simultaneous access to 64 pages, each of which can be in any of the independent address memory subspaces: the system bus, the local bus of any of the computing blocks, and permanent memory of the given block. The logical range of the system bus addressing is $16 \mathrm{M}$, of local bus, it is $1 \mathrm{M}$, and of permanent memory, it is $64 \mathrm{~K}$. Access of each of the processors through the system bus to a local bus of other computing blocks has priority over access from the local processor. Hence, if the processor sends a "lock signal" to the system bus, this blocks access to it from other computing blocks. The correctness of work with commands of the test-and-set type is retained even when they are placed in the local memory.

The ONIKS operating system allowed using several different type processors in "teamwork" mode and provided for "mixed execution" of working mixture for several languages - Yava (Yazyk Vmesto Assemblera, Language instead of Assembler), Cidula (C plus Modula), and Fodula (Forth plus Modula). MRAMOR-based workspaces allowed developing software, creating fonts and preparing publication of a number of newspapers and books, including, for example, a Russian-Chinese phrase book.

\subsection{Physical Considerations}

In one of his last articles, the editors column in the magazine "Mikroprocessornye sredstva I sistemy, No 2, 1988" — "To look ahead, but to see around", Andrei Ershov brought to light the direct parallels between an American forecast list of chief $\mathrm{PC}$ characteristics in the nineties by $\mathrm{W}$. Beem and the features implemented in the MRAMOR workstation. In the process of creating MRAMOR, we paid much attention to the general layout and design of workspaces. Unlike the universally accepted approach, when one places a PC, its display, and other blocks on a desk and occupying most of its surface, the designers of MRAMOR kept in mind the preference for a "large, spacious working desk".

That is the reasons all modules and the FDD are placed in a wheeled side-table; the keyboard is set on a pull-out frame located underneath the desk surface and allowing configuring position and angles. The keyboard had 137 buttons, 134 of them fully pre-programmable; that is, a driver defines their layout and semantics.

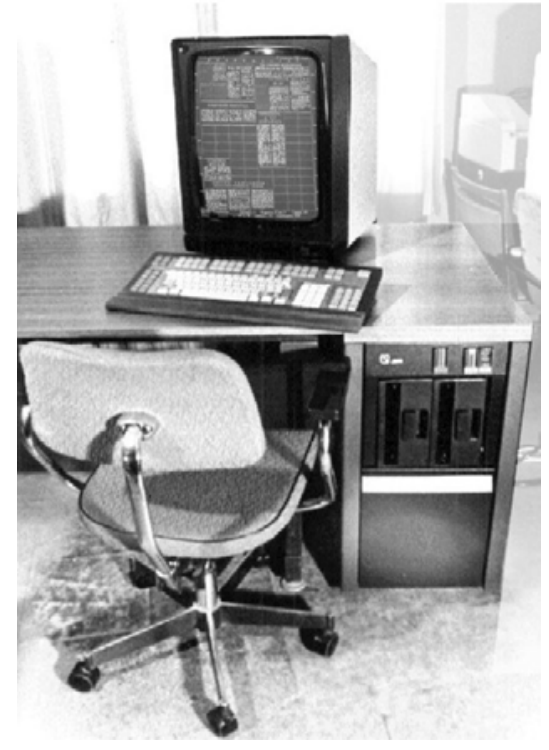

We arranged the buttons on the keyboard into six ergonomic fields, three of which are functional button fields; one corresponds to the typewriter keyboard and the other two for numeric input and cursor manipulation. The five register keys enable working in 32 registers with 128 symbols in each, which allows using as much as 4096 symbols in a single publication. Button fields are color-coded; intervals in which one can put stickers with function captions separated them. There are eight signaling 
LEDs of colors red, yellow, and green with three of them connected to fixed-position buttons.

MRAMOR contained blocks of direct output onto the phototypesetting part of the FA-1000 device and the Gazeta-2 photoreceiving device, which provided quality offset printing forms. MRAMOR also featured software power control because some workspaces could be located in other rooms. Upon finishing a session the user can turn off only his local workspace devices (display and FDD), but not the whole workstation. The basic software did the rest.

Talking of an editor's workspace, the key parameter defining its comfort and usability is the visual quality of textual information, allowing one to display a wide range of symbols in a variety of mark-ups. Hence, we supplied the MRAMOR with a custom-ordered monochrome $50-\mathrm{cm}$ display with high persistence rate to eliminate flickering on the screen with standard TV scanning. Moreover, the declared the use of permanent memory for the generation of symbols as inflexible, and we chose a display scheme featuring support memory. For better correspondence of the image to the format of printing ranges, it was decided that the display be oriented vertically relative to its longer side (the so-called portrait layout, as opposite to the landscape layout seen in most TVs).

At the same time, we retained the standard TV scanning, i.e. vertical, across the text lines. The $64 \mathrm{~Kb}$ memory device of the image allowed creating a 768x576-dot field with three levels of brightness and we hung the display over a table on a special rotating holder enabling variable height and tilt.

\section{The Publication Preparation System}

The MRAMOR WS is a professional system for electronic preparation of polygraph publications of different types and complexity. It provided the publisher with a complete technological cycle from the initial text input to the make-up and production of ready photo forms. We based the system on the principle of separating the text of a publication from its polygraph design. The text itself appears as a double sequence. Its higher level consists of heterogeneous publication elements accessed through a working directory, which also contains types and external characteristics of the elements.

The polygraph design of the publication is coordinated with element types of the publication and/or text mark-up and represented by a separate range-by-range structure. We accomplished the intra-textual

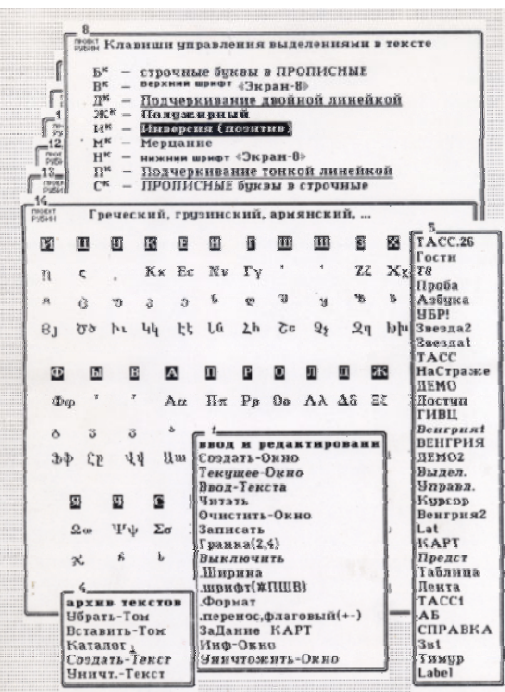
links and footnotes by markers, which are included in the mentioned structures and made the dialogue development of a mechanical and its range-by-range makeup according to the working directory; that is, a system of linked windows support 
editing and correction. We represented fonts by special display fonts; however, we calculated the line formats with real widths of typesetting fonts.

\section{Conclusion}

Our team developed technologies and tools for electronic preparation of publications by means of separating text from its polygraph realization, and possibilities for their separate processing. We developed this with the parallel use of structure-referential representation of the text body combined with text markups for efficient global processing of the text (including the making of mechanicals and make-up). We established the notation of workspaces in different subdivisions of the publishing house, together with the possibility of dynamic transition to functions of different logical workspaces from a single location in the process of work. In the end, our team provided the possibility to prepare a mixed publication in different languages with writing systems within a single workspace. We all felt that this achievement was a true accomplishment.

\section{References}

1. Baehrs, A.A.: Data-processing System RUBIN for "Pravda" newspaper. Applied Approaches in Informatics, 55-78 (1980) (in Russian)

2. Baehrs, A.A.: Software for Typeface Display. Experimental Informatics, 51-80 (1981) (in Russian)

3. Baehrs, A.A., Polyakov, V.G., Rudnev, S.B.: On high-level programming system with mixed computation for personal microprocessor systems. Topical Issues of Computer Architecture and Software, 78-94 (1983) (in Russian)

4. Baehrs, A.A., Polyakov, V.G.: Multifunction workstation architecture for editors' office. Personal Computers in Informatics Problems, 40-49 (1984) (in Russian)

5. Baehrs, A.A., Polyakov, V.G.: Look-and-feel of the system software for editors' office multifunction workstation. Personal Computers in Informatics Problems, 50-57 (1984) (in Russian)

6. Baehrs, A.A.: New-generation workstation MRAMOR. New-Generation Computers Design: Architecture, Software, Intellectualization, 126-141 (1986) (in Russian)

7. Baehrs, A.A.: On object orientation and architecture organization of software systems. Topical Issues of Programming Technology, 4-15 (1989) (in Russian)

8. System analysis of production processes of "Pravda" newspaper publishing. Joint report of Computing Center of SB AS USSR and NB IPMCE, Novosibirsk (1977) (in Russian)

9. Baehrs, A.A.: General scheme of creation and development of data-processing system RUBIN for Pravda newspaper. Pravda Publishing House, Moscow (1979) (in Russian)

Full texts of the documents [8, 9] stored in A.P. Ershov archive are available at http://ershov.iis.nsk.su/ (Software projects section: MRAMOR, RUBIN). 